\title{
COMMENT
}

Check for updates

\section{Health care for homeless people}

Michael Liu ${ }^{1,2}$ and Stephen W. Hwang $\mathbb{1}^{1,3 \times}$

A growing and diversifying homeless population faces tremendous social burdens and structural barriers to health care that contribute to high morbidity and mortality. Health care for homeless people must address intersecting health and social challenges through a combination of health-related and social interventions.

The Organisation for Economic Co-operation and Development (OECD) estimates that 1.9 million people are currently experiencing homelessness in 35 OECD countries (see 2020 OECD Policy Brief). There is no international consensus on the definition of homelessness. In this Comment, we focus on homelessness in high-income regions and on individuals living rough, in emergency accommodation or in accommodation for homeless persons. The global homeless population is growing rapidly owing to increasing urbanization, austerity, income inequality and natural disasters ${ }^{1}$. Moreover, the homeless population is becoming more diverse and now includes a substantial number of youths, women, families, seniors, refugees, and people identifying as Indigenous, racialized and LGBT + . The drivers of homelessness are complex, including structural factors (for example, poverty and lack of affordable housing), systems failures (for example, barriers to health, social and legal services), and individual and relational factors (for example, mental illness and unsafe home circumstances).

\section{Homelessness and health}

Homeless people face intersecting physical, mental and social burdens that greatly increase morbidity and mortality relative to the general population ${ }^{2}$. In Toronto, mortality of homeless men aged 18-64 years was 2-8 times higher than that of non-homeless men $^{3}$; however, it is $\sim 50 \%$ lower than that in some US cities, which may be attributed to universal health insurance and lower homicide rates in Canada, indicative of the strong influence of social institutions on the health of homeless people.

The upstream causes of poor health in the homeless population include extreme poverty, harsh living environments, trauma and structural barriers to care (FIG. 1). The downstream causes include infectious diseases, heart disease, substance use disorders (SUDs) and suicide $^{2}$. Mental illnesses and SUDs are quite prevalent among the homeless population; for example, alcohol and drug dependence rates are $\sim 38 \%$ and $\sim 24 \%$, respectively ${ }^{4}$. Other important health-related issues are poorly controlled chronic diseases, high prevalence of traumatic brain injury, disproportionate victimization and high rates of tobacco use ${ }^{2}$. Over time, medical causes of mortality have changed. The standardized mortality rate of homeless adults in Boston remained the same in 1988-1993 and 2003-2008 (REF. ${ }^{5}$ ). During these periods, deaths due to drug overdoses, psychoactive SUDs and suicides increased by twofold to threefold, but deaths caused by HIV/AIDS and cirrhosis declined.

The COVID-19 pandemic further threatens the health of homeless people, who are at higher risk of contracting SARS-CoV-2 and developing severe disease ${ }^{6}$. Homeless shelters facilitate transmission owing to shared living quarters, crowding and high population turnover, and common health problems in this population (respiratory conditions, heart disease and tobacco use) are risk factors for severe COVID-19 disease.

\section{Challenges in homeless health care}

Homeless people must overcome tremendous structural barriers to obtain health care, including lack of health insurance and financial difficulties. They must also contend with competing priorities, such as securing food and shelter, which frequently take precedence over health care. Homeless people may also avoid care owing to a mistrust of the health-care system and experiences of discrimination from providers.

Primary care is essential to improving the health of this population, owing to its longitudinal and relationshipbased nature. Its providers are well-positioned to identify social contributors to poor health (for example, income and food insecurity) and connect patients to community-based services ${ }^{1}$. Unfortunately, many homeless people lack a source of primary care, and their care pathways often rely on acute services ${ }^{7}$. Regardless of universal health insurance, rates of emergency department visits and hospitalizations are much higher in the homeless population than the general population ${ }^{2}$.

Also, concerning evidence shows that care provided to homeless patients is less appropriate than that provided to others. For example, homeless adults hospitalized with acute myocardial infarction in three US states were less likely to receive life-saving diagnostic and therapeutic procedures than non-homeless adults ${ }^{8}$. Moreover, hospital discharge planning for this population is suboptimal and contributes to high re-admission rates ${ }^{9}$. 


\section{Causes of morbidity and mortality}

Health-care and social interventions

Upstream causes

- Poverty

- Trauma throughout lifecourse

- Victimization

- Discrimination

- Social exclusion

- Experience of homelessness

- Harsh living environments

- Poor nutrition

- Competing priorities

- Barriers to health care

- Mistrust of health-care system

- Medication non-adherance

$\downarrow$
Downstream causes
- Heart disease
- Respiratory conditions
- Liver disease
- Infectious diseases
- Musculoskeletal diseases
- Mental illness
- Substance use disorders
- Uncontrolled chronic diseases
- Suicides, homicides and
accidents
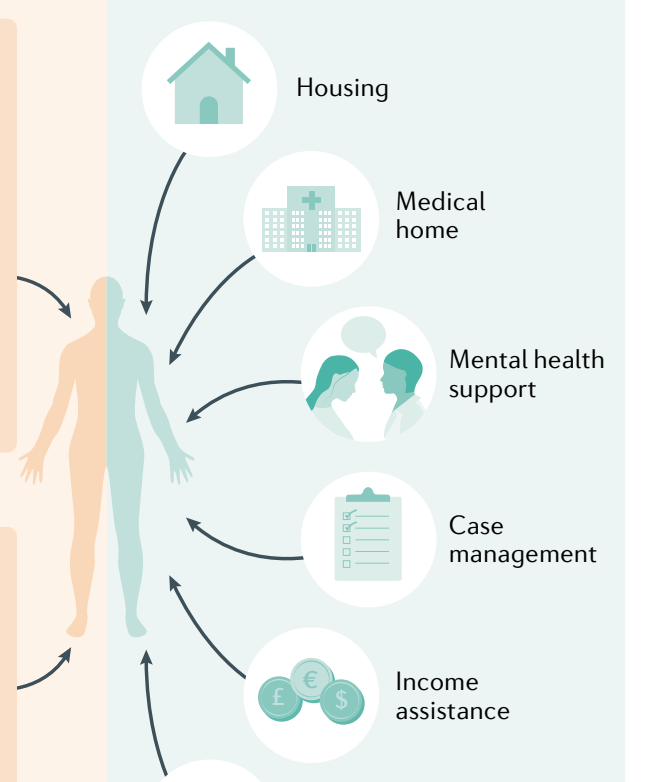

Case management

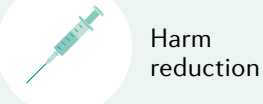

Fig. 1 | Interventions to improve health outcomes among homeless people. Upstream systemic barriers and burdens contribute to downstream medical causes of high morbidity and mortality of homeless people. In turn, downstream causes magnify negative effects of upstream causes. Thus, care for homeless people must address intersecting health and social burdens by combining health-related and social interventions.

The COVID-19 pandemic and resultant closures have exacerbated barriers to care and services, likely increasing overdoses and other health problems in the homeless population.

\section{Approaches in homeless health care}

Care providers for homeless patients should champion three priorities: delivering trauma-informed and person-centred care, linking patients to comprehensive primary care and engaging interdisciplinary community-based services ${ }^{1}$. Traditional health care must be combined with efforts to address social determinants of health through culturally appropriate services, including housing, case management (CM) and harm reduction.

Health, health care and housing are inextricably linked. All homeless patients should be connected to services that assist with obtaining housing. For those with high needs, permanent supportive housing (PSH; long-term housing with individualized support) should be considered. Several studies have shown that PSH provided without preconditions results in rapid and stable housing and positive effects on health and health service use ${ }^{1}$. Housing interventions are frequently deployed alongside CM, a coordinated approach to supportive care. The effectiveness of CM programmes for improving homeless health is mixed and varies across programme models and patient subgroup $\mathrm{s}^{10}$. Income-assistance interventions improve housing stability, although their effects on health and health service use are unclear ${ }^{1}$. Notably, many interventions such as PSH and CM are

cost-effective, given downstream savings in medical and social services ${ }^{1}$.

Additional interventions are recommended for homeless patients with specific needs. For those with complex needs and/or mental illness, care providers should connect patients with community mental health programmes, $\mathrm{CM}$ and psychiatric services. Those with an opioid SUD should be provided with naloxone kits and referred to opioid agonist therapy ${ }^{1}$. Homeless patients with other SUDs should be referred to addiction and harm reduction services. Supervised consumption facilities reduce opioid overdoses, decrease syringe sharing and encourage initiation of methadone maintenance therapy ${ }^{1}$.

\section{Next steps for homeless health care}

Health care and community service providers must collaborate to meet the unique needs of the homeless population. Providers should foster trust and sustained relationships with homeless people. Health systems must facilitate continuity of care, ensure primary care and communitybased services are accessible, and continuously monitor and improve quality of care for this population.

The provision of housing is important for homeless health. COVID-19 has eroded health in this population, but it has demonstrated that the homelessness crisis is indeed solvable with enough political will. During the pandemic, the USA, UK, Australia, Canada and others have housed an unprecedented number of people in hotels and temporary facilities. However, housing alone is insufficient. Previous research has focused almost exclusively on housing or health-care interventions for this population. Yet, homelessness is a multifaceted problem that requires a combination of housing, health-care and community-based solutions. Interdisciplinary collaborations between health systems and social services are needed to end homelessness and improve health and well-being for this population.

For further information, please see Institute of Global Homelessness, FEANTSA, National Health Care for the Homeless Council, Homeless Hub.

1. Pottie, K. et al. Clinical guideline for homeless and vulnerably housed people, and people with lived homelessness experience CMAJ 192, E240-E254 (2020).

2. Fazel, S., Geddes, J. R. \& Kushel, M. The health of homeless people in high-income countries: descriptive epidemiology, health consequences, and clinical and policy recommendations. Lancet 384, 1529-1540 (2014)

3. Hwang, S. W. Mortality among men using homeless shelters in Toronto, Ontario. JAMA 283, 2152-2157 (2000).

4. Fazel, S., Khosla, V., Doll, H. \& Geddes, J. The prevalence of mental disorders among the homeless in Western countries: systematic review and meta-regression analysis. PLoS Med. 5, e225 (2008).

5. Baggett, T. P. et al. Mortality among homeless adults in Boston: shifts in causes of death over a 15-year period. JAMA Intern Med. 173, 189-195 (2013).

6. Tsai, J. \& Wilson, M. COVID-19: a potential public health problem for homeless populations. Lancet Public Health 5, e186-e187 (2020).

7. Khandor, E. et al. Access to primary health care among homeless adults in Toronto, Canada: results from the street health survey. Open Med. 5, 94-103 (2011).

8. Wadhera, R. K. et al. Disparities in care and mortality among homeless adults hospitalized for cardiovascular conditions. JAMA Intern Med. 180, 357-366 (2020).

9. Miyawaki, A., Hasegawa, K., Figueroa, J. F. \& Tsugawa, Y. Hospital readmission and emergency department revisits of homeless patients treated at homeless-serving hospitals in the usa: observational study. J. Gen . Intern. Med. 35, 2560-2568 (2020).

10. de Vet, R. et al. Effectiveness of case management for homeless persons: a systematic review. Am. J. Public Health 103, e13-e26 (2013).

\section{Competing interests}

The authors declare no competing interests. 